\title{
A message from the new editors-in-chief of Energy Systems
}

\author{
Panos M. Pardalos ${ }^{1}$ - Qipeng P. Zheng ${ }^{2}$
}

Published online: 19 February 2018

(C) Springer-Verlag GmbH Germany, part of Springer Nature 2018

The journal Energy Systems (ENSY) publishes papers on mathematical programming, control, and economic approaches towards energy systems related topics. We focus on papers that deal with theoretical, computational, and applied aspects of energy systems as well as state of the art expository/review papers. In particular, we publish research related to the major worldwide energy challenges confronting humanity in this century. There is no doubt that energy is an important part of national security for every country in the world. Smart manufacturing, also known as the fourth industrial revolution, would not have occurred without advances in energy systems. In addition, the rapid growth of energy consumption within existing infrastructures is incompatible with the sustainable development of mankind. Interdisciplinary research on energy systems has grown dramatically as scientists try to address many of the technical problems related to energy.

The first issue of Energy Systems was published in 2010 under the leadership of the journal's founding editor, Panos Pardalos, who managed the journal with the help of an active editorial board, until 2016. In 2016 Steffen Rebennack joined the editorial staff as co-editor and in 2017 became the editor-in-chief of ENSY. We would like to

$\triangle$ Panos M. Pardalos

p.m.pardalos@gmail.com

$\bowtie$ Qipeng P. Zheng

qipeng.zheng@gmail.com

1 Department of Industrial and Systems Engineering, University of Florida, Gainesville, Florida, USA

2 Department of Industrial Engineering and Management Systems, University of Central Florida, Orlando, Florida, USA 
thank Steffen for his expertise and hard work as editor in helping the journal grow and become well-recognized by the scientific energy community.

With this first issue of 2018 we will serve as co-editors-in-chief of ENSY. With so many diverse and excellent journals related to energy, scientists have a wide selection of journals to publish their results. We need to emphasize the uniqueness of ENSY for the authors to select our journal for publishing their results based on the following rationales:

- We can accept short papers and publish them on time.

- We will continue our efforts to select referees who are experts on the subject of each submitted paper.

- We plan to have special issues on hot developments in Energy System (for example a special issues on Blockchain technologies is planned).

- We plan to increase the visibility of the journal in the scientific community at an international level.

Success is not based only on ideas but mostly on execution. That is why we rely on the help and advice of all members of the editorial board to establish ENSY as one of the leading journals in energy research.

We would like to take this opportunity to thank Christian Rauscher of Springer for helping us with the journal, all members of the editorial board of ENSY, and all authors and reviewers for their enthusiasm and hard work. We look forward to serving Energy Systems and for its continuing success as a leading energy journal. 